reduction. Reduction and alignment are shown by radiograph to be almost anatomic (Fig. 10), Convalescence was uneventful. Union occurred by first intention. There was no shortening.

CASE 5.-Man, aged 32. Comminuted fracture of left humerus, with marked displacement (Fig. 11). There was a large hematoma extending into the axilla and on the chest anteriorly and posteriorly. Operation was done nine days after the injury. I considered it safe to wait until the tissues had gained some resistance. Several fragments of bone were removed at the operation. Their absence is well shown in the radiograph (Fig. 12). Convalescence was uneventful, Union by first intention took place. The patient undressed and dressed himself without assistance thirty-two days after the operation.

CAsE 6.-Boy, aged 18. Compound comminuted fracture of the left humerus, caused by passage of two wheels of a loaded coal wagon $(7,450$ pounds) over the member. Operation was performed two days after the injury. The patient traveled 150 miles from an inaccessible part of the state. The comminuted fragments were removed. The extensor muscles of the arm were severed by the crushing force of the wheels. The musculospiral nerve was tucked into the lower segment of the triceps. There was no infection of wound, which healed kindly as soon as the devitalized soft parts separated. Alignment is well shown in the radiograph (Fig. 13). There was some paresis of the flexors and extensors of the forearm, but at the time of the last report (nine weeks after the injury) power was rapidly returning.

1723 Tremont Place.

\section{A METHOD OF TONSILLECTOMY BY MEANS OF A GUILLOTINE AND THE ALVEO- LAR EMINENCE OF THE MANDIBLE}

\section{GREENFIELD SLUDER, M.D.}

Clinical Professor for Diseases of the Nose and Throat, Medical Department of Washington University ST. LOUIS

I wish to describe a method of tonsillectomy which, so far as I can learn, has not heretofore been described or used. The essential and distinctive feature of this method is the fact that it moves the tonsil completely out of its normal bed in the forward and upward direction and then utilizes one of the anatomic markings of the lower jaw as a vantage-point in putting it through the aperture of the guillotine. This anatomic marking is the well-defined eminence just above the mylohyoid line, produced by the last-formed molar tooth in its socket (Fig. 1, a, Fig. 2, I, a, Fig. 2, II, a), which is rendered even more prominent in the mouth by the tissues of the gum. In childhood the posterior, unformed molar as it lies imbedded in the alveolus helps to make the eminence (Fig. 2, II, a). This marking has not been given a name in any of the treatises on anatomy. I have taken the liberty of naming it "the alveolar eminence of the mandible," in order to facilitate the description of this procedure.

The instrument used (Fig. 3, I and 3, II) is a modification of what I have nearly always heard called the "Mackenzie guillotine"; but it occasionally has been called the "English guillotine" to distinguish it from the "Mathieu" or "French guillotine." Sir Morell Mackenzie, ${ }^{1}$ however, relates that the instrument which nowadays bears his name is a modification of the one described by Dr. Physick ${ }^{2}$ of Philadelphia in 1827, the difference being that he (Mackenzie) made the handle

1. Mackenzie, Morell : Diseases of the Pharynx, Larynx and Trachea, New York, Wm. Wood \& Co., 1880.

2. Physick : Am. Jour. Med. Sc., i, 262, Quoted bs Mackenzit. reversible, so that it could be applied to either side of the shaft. In this way he facilitated its use by surgeons who used the one hand for removing both tonsils. He also narrates that Dr. W. B. Fahnestock ${ }^{3}$ of Lancaster, Pa., in 1832, first described the modification which consists of a circular aperture and a transfixing needle, with a ring. knife to cut on the pull (the

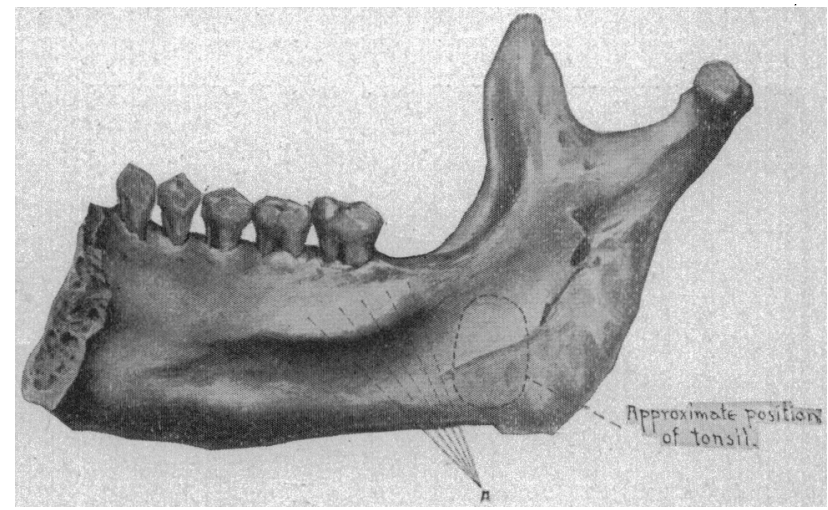

Fig. 1.-Inner surface of the right half of the mandible, showing the alveolar eminence, $A$, and its relation to the usual position of the tonsil.

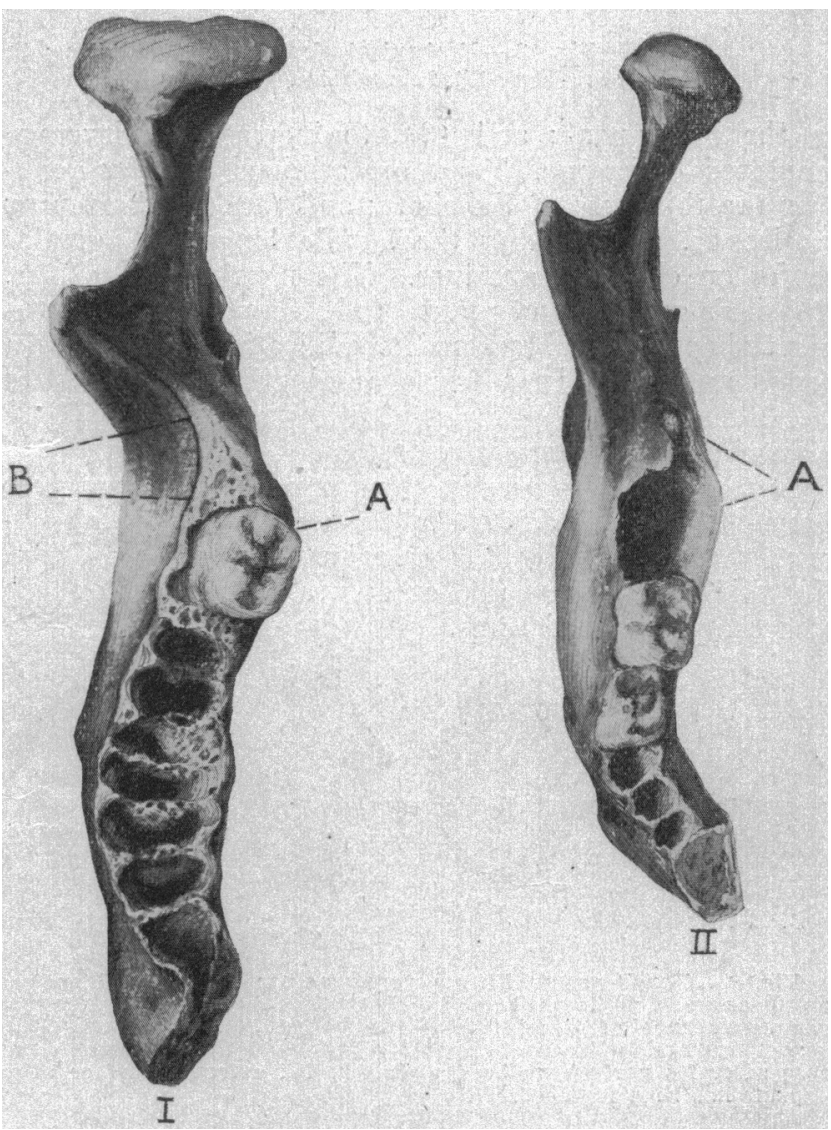

F:g. 2.-I. Right half of a mature mandtble seen from above, showing alveolar eminence, $A$, with fully cut third molar and buccinator ridge $B$. II. Right half of young mandivle seen trom above, showing uncut third molar and the part it takes in the formation of the alveolar eminence $A$.

"French guillotine") and that Guersant" in 1864 changed the shape to an ellipse and at the suggestion of Velpeau added the elevating prongs. He furthermore states that the guillotine idea was first published

3. Fahnestock, W. B.: Am. Jour. Med. Sc., xi, 248. Quoted by Mackenzle.

4. Guersant: Hypertrophle des amygdales, Paris, 1864. Quoted by Mackenzie. 
by Dr. Benjamin Be!l ${ }^{5}$ in 1783 in his description of the uvulatomes used in England in the latter part of the eighteenth century.

The guillotine which I use (Fig. 3, I and II) is therefore a modification of that of Dr. Physick, which consisted of a shaft of metal with a circular aperture at its distal end through which the tonsil was inserted, and cut through by a blade pushed across the aperture. I have altered the original model in order to accom. plish two definite objects: (1) to get greater strength

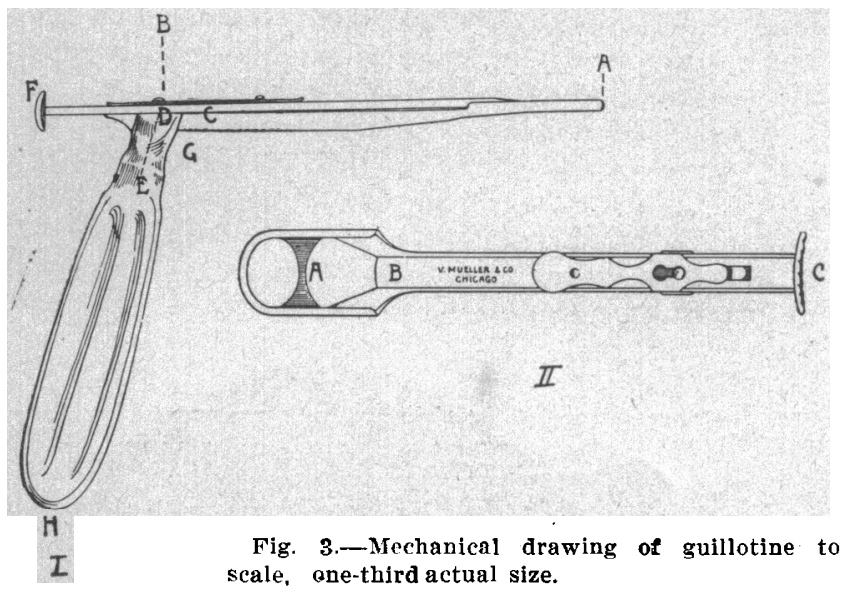

in the instrument; and (2), to increase the leverage employed in its use. These are attained (1), by doubling the thickness of the shaft; and (2), by shortening its length, and making the handle longer. I greatly prefer an elliptical aperture. The usual model of Dr. Physick's guillotine at present to be found in the instrument shops is the Mackenzie modification, which has a round aperture. In a few shops in the United States it has been made with an elliptical aperture the long axis of which is transverse to that of the shaft.

In this operation a correct instrument is of very great importance. I believe it to be of far greater importance than is usually the case in surgical procedures. For this reason $I$ feel that an accurate description ought to accompany its pictures.
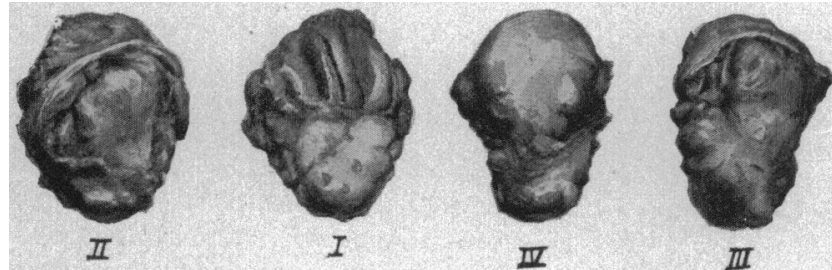

Fig. 4.--Tonsil removed from young adult: I, internal surface as it appears while still in the grasp of the guillotine, having the appearance of being turned inside out; II, same tonsil, external surface, with the capsule invaginated; III, internal surface after its parts have been replaced; IV, external surface after capsule has been replaced.

\section{DESCRIPTION OF THE GUILLOTINE}

(In the following use of terms I assume that the surgeon is holding the instrument, with its aperture from him). It is made entirely of metal. It measures 13. $\mathrm{cm}$. from $A$ to $B$ (Fig. 3, I) which is from 2 to 4 $\mathrm{cm}$. shorter than the models usually found for sale. The angle at which the shaft and handle join, $C, D, E$, is $115^{\circ}$. This is the most comfortable position for the handle in the surgeon's hand; and sacrifices almost

5. Bell, Benjamin: System of Surgery, 1783, iv, 144, Plate 52, Fig. 1. Quoted by Mackenzie. none of its leverage. The handle should be large and rough, measuring $11.5 \mathrm{~cm}$. from its free end $H$ to its junction with the shaft $G$; and should be of a shape that is comfortable in the grasp, thus facilitating the use of strength. The shaft is thin, $3 \mathrm{~mm}$., at its distal end where the aperture is cut; but is reinforced on the handle side, proximal to the aperture, by another piece of steel of the same or greater thickness, they being welded together and the handle attached to these. Special attention should be paid to details in order to make the instrument as strong as possible, such as putting a bolt through the full length of the handle in addition to its being welded to the shaft. Its strength should be so great that the shaft cannot be bent or the handle broken off by the power of an ordinary man. The blade is $2.5 \mathrm{~mm}$. thick. Its proximal end, $\dot{F}$, when closed (Fig. 3, I, is drawn with the blade open) should extend $2.5 \mathrm{~cm}$. beyond the distal side of the handle at its attachment to the shaft $G$. Its cutting edge should be made dull for the reason that it will then readily follow in the connective tissue between the capsule of the tonsil and the constrictor of the pharynx on which it lies, whereas a sharp edge easily cuts through the tonsil or the constrictor. Less bleeding follows from the wound of a dull blade, which is an additional advantage. The edge should be made by grinding the blade $2.25 \mathrm{~cm}$. back (Fig. 3, 11, $A, B$ ), because by so thinning the cutting end of the blade the field of operation is

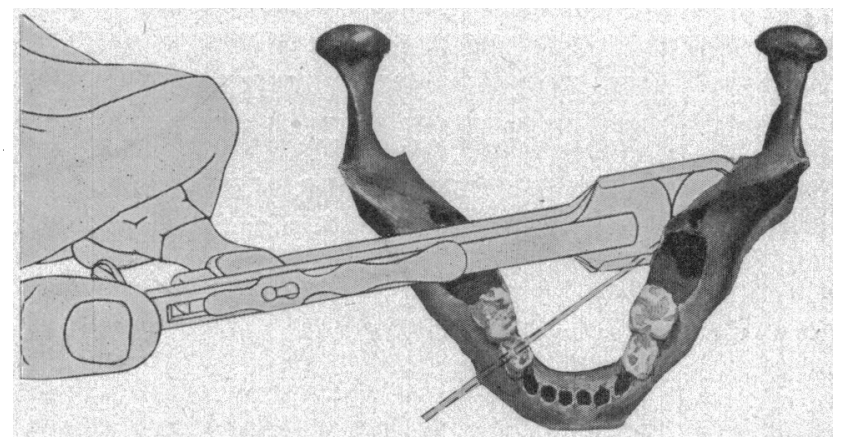

Fig. 5.-A young faw showing gulliotine in positton. The dotted lines show the usual position of guillotine for mature jaws.

made wider, as will be shown later. The proximal end of the blade is provided with a cross-bar $F$, which should be comfortable under the thumb so as to permit of great pressure without hurting the surgeon's hand. A ring may be substituted for the cross-bar and the surgeon's thumb inserted through it. The assumed advantage in this is that, in case he should not apply the instrument perfectly, he could readily withdraw the blade by means of his thumb without removing the instrument from its position, and immediately push it down again. This might be repeated until he had the tonsil properly through the aperture, with small loss of time; but he soon learns to do this with the cross-bar too, so at present my feeling is that this must be a matter of individual choice.

The blade must slide to and fro with the least possible friction. The aperture is elliptical, with its long axis transverse to that of the shaft. Its distal arc should be lined around with a soft metal into which the dull blade may be forced in the act of cutting through the tissues. The surgeon should have two guillotines, one with an aperture $2.5 \mathrm{~cm}$. by $1.8 \mathrm{~cm}$., the other 1.75 $\mathrm{cm}$. by $1.5 \mathrm{~cm}$. The two instruments should be alike in every measurement except the distal part of the shaft 
which bears the aperture; the two should be of the same great strength. If the shaft is not strong enough it will be bent slightly in the act of operating and then the blade will not slide with the ease that is required of this instrument. If the handle is not securely attached to the shaft it will be broken off during the operation.

\section{ANATOMIC RELATIONS}

In the following use of terms indicating direction I assume the body of the patient to be erect and facing the surgeon. I have already mentioned that one of the essential and distinctive features of this method is the utilization of the alveolar eminence of the mandible in manipulating the tonsil. I have also stated that this eminence is made by the internal ending of the alveolus, with the gum covering it.

It should be remembered that the tonsil lies posterior to and for the most part below the eminence, in childhood appearing much further back and much lower than in maturity. This arises from the fact that an uncut tooth is in the alveolus posterior to the one showing through the gum membrane, causing a greater elevation at this site and making it seem that the teeth are much further forward, and the tonsil lower. In cases of great enlargement of the tonsil more of it will lie even with or a part may possibly lie above the eminence. It will also extend forward, approaching the eminence from behind.

The surface of the jaw-bone posterior to and below the eminence slants downward, backward and outward at an angle that may vary considerably, but is approximately $45^{\circ}$ - that is $45^{\circ}$ downward and outward from the transverse, and at the same time $45^{\circ}$ outward and backward from the anteroposterior axis. This slant arises in large part from the fact that the alveolar arch as it proceeds backward tilts more and more inward from the body of the jaw. Its posterior end faces inward as well as upward (Fig. 2, I $A$ ). Looking backward or downward it is seen that the ending of the alveolus lies completely internal to the line of the buccinator ridge were it prolonged as it descends on the coronoid process (Fig. 2, I B); and the impression thus given is that this line when carried downward represents the jaw proper and that the end of the alveolus lies internal to it. This is shown better on mature jaws. This slant is crossed by the mylohyoid line, which is well marked in maturity. On young jaws this surface is more nearlv smooth, although that marking is always to be seen.

The angle of this slant is subject to considerable variation because the relation of the breadth of the alveolus to the height of the horizontal portion of the jaw (alveolu's and body together) varies very much according to age. The breadth of the alveolus in the vouno is actually wider ( $3 \mathrm{~mm}$. sometimes) than that of the adult. This is true for two reasons; (1) that in the young, these alveolar dimensions are made by the crown of the molar, whereas in maturity they are made by its neck; (2) that although the tooth may be through, the bone surrounding it has not yet been absorbed to the extent that it is in maturity. On the other hand the height of the horizontal portion in maturity is twice that of early life, for the reasons that the young jaw consists largely of alveolus, and that the body is added to it only by development with the years. For these reasons the angle of the slant in young jaws may rise to even more than $60^{\circ}$ outward and backward from the anteroposterior axis (Fig. 5), and to even more than $60^{\circ}$ downward and outward from the transverse; while in maturity these angles may be less than $45^{\circ}$ in one or both directions. The alveolar eminence is therefore very much more prominent in the young, despite the fact that the inward tilting of the alveolus is greater in maturity than in early life. The edentulous jaw need scarce be considered in this connection, because the operation in question is rarely needed by the aged. Anatomically, however, it is usually marked by a very heavy mylohyoid line which, since the absorption of the alveolus, lies near the upper margin of the body which alone remains. There is usually a trace of the alveolar eminence still to be observed.

These differences may be accurately estimated in the living by introducing the index-finger into the patient's mouth, downward, outward and backward from the alveolar eminence pushing the soft tissues aside as far as may be necessary. It is distinctly advantageous to make this estimate just before operating in order that the direction of the guillotine may be varied to suit that individual's requirements. The buccinator ridge is frequently helpful in making this estimate.

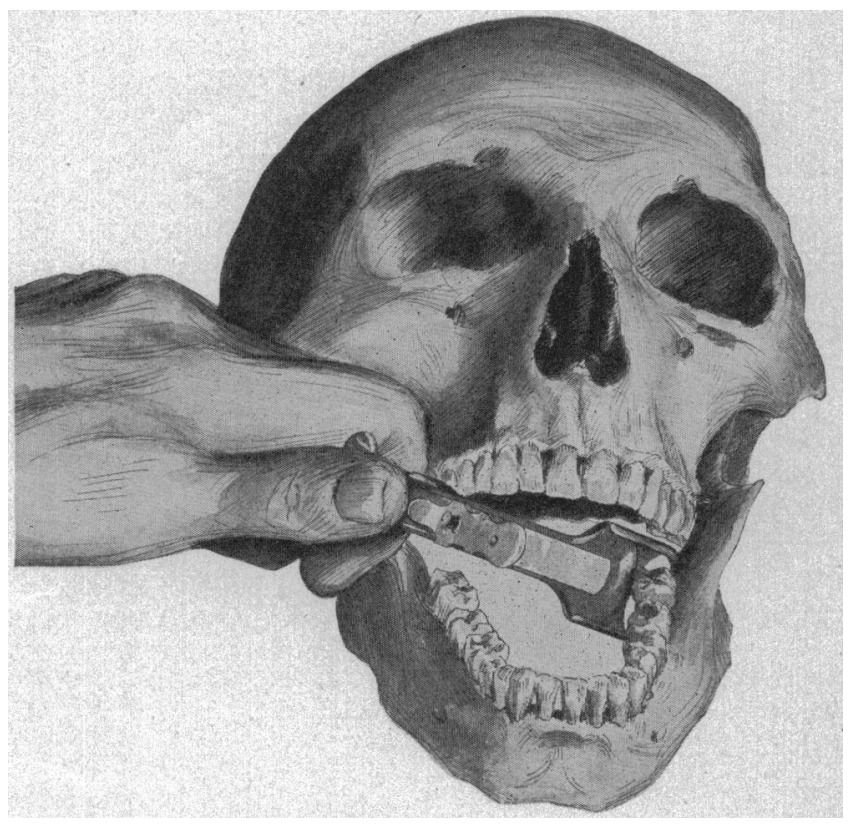

Fig. 6.-The guillotine in position, showing its general relationship to the bones.

\section{THE OPERATION}

In order to use the alveolar eminence of the mandible as a vantage-point from which to manipulate the tonsil - that is, to use this prominence to put or help to put the tonsil through the aperture of the guillotine-it is necessary to move it completely from its normal position which is posterior to and below the eminence (see above). It must be moved forward and upward. The elasticity of the soft parts of the throat readily allow the necessary movement. In this way the tonsil will be moved out of a hollow, soft, moving bed and be brought up on to a motionless, hard hump-a solid. fixed, somewhat hemispherical convexity.

Under these altered conditions it is not difficult to place the blade of the guillotine at its base. The eminence is then sometimes found to be all that is required to hold it for a perfect adjustment of the instrument, which is done by pressing it against the bone. The prominence of the eminence then stuffs the tonsil through the aperture. Often, however, in operating, especially with a large, fiat (thin) imbedded tonsil, it is 
necessary to use the tip of the index-finger of the other hand to put the final bit through. If it be soft in addition to these qualities, the difficulty is increased to the fullest possibility. A firm tonsil is manipulated without difficulty, regardless of size or imbedding, provided it be not too thin (flat). Should it be soft, large, flat (thin) and imbedded, great care and some dexterity must be exercised in order to secure it entire.

Under these conditions the surgeon will find sometimes that he has removed only the central portion of the capsule with tonsil tissue surrounding it. A little more familiarity with the method and possibly a little greater dexterity in managing the instrument will usually permit him to secure it entire. (I can, however, picture to myself a tonsil that is so soft, thin and spread out that it could not be removed entire with one stroke, despite all the care and dexterity that might be exercised; but it must needs be rare.)

Under conditions presenting difficulties a perfently satisfactory guillotine is indisnensable. Precise knowledge of the topography and all the variations is necessary at all times.

\section{THE OPERATIVE PROCEDURE}

The use of this type of instrument, so far as I am able to learn, has always been by preference with the smooth (proximal) side of the blade applied to the tonsil. Mackenzie ${ }^{1}$ made his modification with its detachable and reversible handle that this might be done more readily (see above). Its method of use was, that it was inserted into the mouth in an anteroposterior direction and the aperture put over that part of the tonsil which protruded beyond its surroundings into the cavity of the throat. The blade was then pushed across, cutting off that portion.

With my method one must approach the tonsil at an angle, approximately $45^{\circ}$, which requires the shaft of the instrument to cross the mouth entirely from the opposite side. This necessitates the distal side of the shalft being applied to the tonsil. It at the same time has the great advantage of leaving the lateral portion of field of operation wide open for view and the use of the fingers of the other hand. Having the blade ground far back. widens it further. ('The use of the proximal side will be found exceedingly awkward or even impossible as the surgeon's hands are interfered with and the lateral portion of the field of operation covered from view.)

It is a very great advantage to operate on the right tonsil by holding the guillotine in the right hand and on the left tonsil by holding it in the left hand. If the surgeon can use only one hand, the position of the patient should be recumbent. Assuming that he uses his right hand for both tonsils and stands on the patient's right: for the right.one he faces the patient's head; hut for the left one he must turn around so that he faces the patient's feet, and stand somewhat bevond (above) his head. (These relations might be sustained in the erect posture also.) The head should be held firmly by an assistant to prevent rotation, and the mouth kept fully open by a gag.

Regardless of what may be the position of the patient's head, the surgeon takes his bearings from the lower jaw. The guillotine, with the transverse axis of the aperture vertical, is introduced into the mouth at an angle of $45^{\circ}$ outward and backward, passing back until the dictal are of the aperture is completely behind the tonsil. The lirection of the shaft is then changed to noint downward in order to get the ring of the aperture under the lower- most part of the tonsil. The instrument at this moment may sometimes to advantage be rotated slightly by turning the handle downward (toward the feet). This tends to enlarge the field of vision. It is then pressed outward until the distal arc of the aperture has been pressed" against the ramus of the jaw, or, in case the patient is not anesthetized, against the firmly contracted internaI pterygoid muscle which is inserted here. It is now brought slightly forward and upward, but held firmly against the bone and muscle, when it will be seen that the lower distal arc of the aperture has acted very much like a scoop, having secured the lower part of the tonsil and brought it forward and upward into the neighborhood of the alveolar eminence. In case the shaft has been rotated to secure its lower part, it is now put back into its original position by turning the handle upward. The upper portion of the tonsil is usually put into the grasp of the distal arc of the aperture by this rotation. If the tonsil is not too large and flat (thin) it is usually secured, both lower and upper portions, in the first setting of the guillotine and no rotation is needed. The distal arc of the aperture is now firmly held behind the posterior border of the tonsil and the instrument drawn forward and upward at an angle approximately $45^{\circ}$, which will be found to have pulled it upward and forward onto the eminence of the alveolus. The blade is now pushed down with the gentlest possible pressure until the surgeon sees that it is in contact with the tissues. It should not be pressed forcibly until the parts are engaged satisfactorily in the aperture. The blade. being in contact with the tissues, prevents the portion of tonsil which has gone through from slipping out again.

At this moment the surgeon may perceive that although the distal are of the aperture is entirely behind and external to the tonsil, a part of its anterior portion has still not gone through. This is usually readily seen. but may be more definitely determined by feeling with the tip of the index-finger of the other hand; and at the same time it may be pushed through. This is done by the gentlest massage-simply stroking it in the direction of the aperture with the ball of the index-finger and, at the same time, pushing the blade very gently across the remaining portion of the aperture. When the last of the anterior portion has gone through it may be readily seen as a rule; but may be determined with great accuracy by feeling with the ball of the index-finger-passing it up and down over the end of the blade as it closes the aperture. If all of the tonsil has gone through, the distal are will be felt smonth and firm and to be covered by what seems to be a thick mucous membrane. (It is of course two layers of membrane.) If a part of the tonsil has not gone through, it can easily be felt and recognized as a mass of tissue harder than membrane. and usually irregular.

All of the tonsil having gone through, the blade is now pushed across with all the power of the surgeon's hands. Great pressure is usually required because the blade has been made dull. If it be too dull or if it does not fit perfectly into the soft metal lining of the distal are of the aperture, it will not cut altogether through. The instrument must then be pulled forward a little and its end be stripped off with the finger of the other hand. This does not delay the operation. It may possibly make a little more subsequent pain; but I am by no means sure that it does.

On examination of the extirpated tonsil while it is still held in the grasp of the instrument--that is, just as it comes from the patient's mouth-it will be seen 
that its surface which was exposed in the throat has the appearance of having been turned inside out (Fig. 4,I). On loosening it from the instrument it is seen that its capsule side-its lateral surface-bears further proof of this fact. The capsule appears completely invaginated (Fig. 4, II) ; but it can be readily replaced-that is, turned over into its original relations, thereby restoring the tonsil to its customary appearance, as it is in the throat (Fig. 4, III), covered on its lateral, convex aspect by the capsule (Fig. 4, IV). This phenomenon is readily explicable when one remembers that it was brought up on to the eminence to have the ring of the guillotine, as it were, stuffed down over it, converting its convex bed into a concave one at the time of cutting. Further examination of the specimen reveals that a little of the free edge of the anterior pillar, including a few fibers of the palatoglossus muscle, is attached to it. I always remove this bit of the anterior pillar with the tonsil because it leaves a more open wound and a more open fossa when healed. There seems to be no surgical reason against it; on the contrary, it makes the tension of the scar begin at a point anterior to and below what would otherwise be the case, which tends to draw the palate forward and downward. In cases in which the tonsil is to be removed because of Eustachian tube irritation this seems to me to be advantageous. In operating under general anesthesia I prefer to have the pharyngeal reflex present. I much prefer to have the aperture fit rather snugly over the tonsil.

This method will be found to be satisfactory under all usual conditions, provided the variations in the jaws of different ages are borne in mind. For the young jaw the angle of the instrument must sometimes be increased to even more than $60^{\circ}$ outward and backward and to even more than $60^{\circ}$ downward and outward. It does not require grasping the tonsil in a vulsellum and pulling it out. The imbedded tonsil is usually removed as easily and as quickly as the protruding one (see above). No loosening of the pillars is required, because I prefer to take a little of the anterior one. Familiarity with the method will enable the surgeon to remove a tonsil in its capsule complete in from five to eight seconds. I think that the shock from this method is less than from the longer operations.

Stumps of tonsils left from previous operations are usually as easily removed as the original tonsils.

3542 Washington Avenue.

Intestinal Sutures.-The evolution of the intestinal suture has brought us to the point where we are certain of good results when the sutures are properly applied and are of the right material. Non-absorbable suture material should be cast off into the lumen of the intestine after serving its period of usefulness. This is best accomplished when used for the inner layer. There is also less destruction of tissue. When placed externally it promotes unnecessary adhesions, plastic exudate and fibrous bands, and may interfere with the proper bowel function becalise of its prolonged irritation. Absorbable suture material used for the outer layer is soon taken care of, and does not cripple the action of the intestine. The suture in intestinal work must control hemorrhage, secure proper coaptation of the tissues, prevent leakage, avoid pursing, be quickly introduced and must not strangulate the tissue. The back lockstitch of Pagenstecher's thread serves all these purposes. Experimental work has demonstrated that chromic catgut when employed as the outer suture lasts s"finiently long for positive and firm union to occur.-A. E. Benjamin, in the Lancet-Clinic

\section{LIPOMATOSIS OF THE LOWER EXTREMITIES} WITH REPORT OF A CASE

W. R. MacAUSLAND, M.D.

Orthopedic Surgeon, Carney Hospital

$$
\text { AND }
$$

B. E. WOOD, M.D.

Assistant Orthopedic Surgeon, Carney Hospital BOSTON

This case of lipomatosis of the lower extremities is reported on account of its rarity. Its steady progression suggested a malignant process. No literature is found bearing on this subject.

Patient.-R. R., a girl aged 10, was seen in consultation with Dr. B. L. Dwinell of Taunton, Mass., Dec. 8, 1907. She had suffered since birth with slowly progressing deformity of feet. The family history was negative. "Both feet at birth were shaped like animals' feet, and much larger than normal in pro-

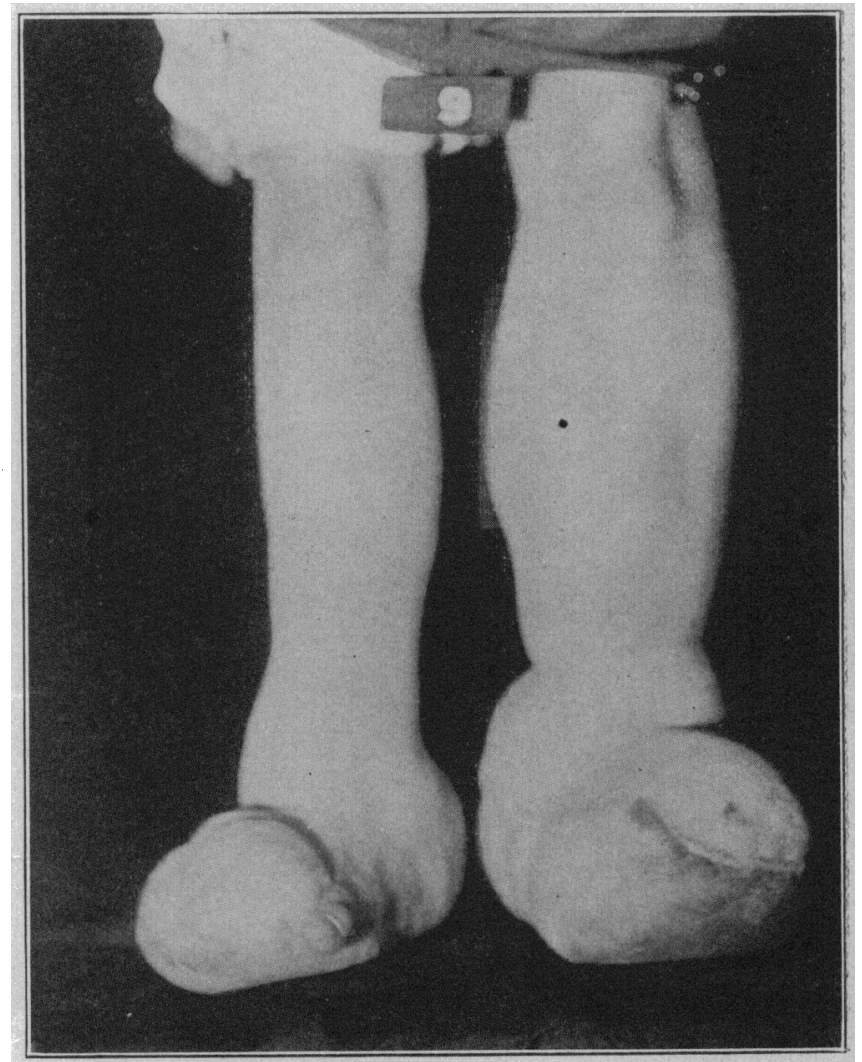

Fig. 1.-Lipomatosis of feet and legs ; front view.

portion to the body; weight at birth, $4 \%$ pounds. Patient had on left side a peculiar mark, which locked like an old burn: otherwise had been normal and healthy. The patient's mother was not as well as usual when carrying the child, and lad a great deal of pain at night. At the age of 6 months, the child's toes were amputated. At this age the feet were noted to be growing faster than the rest of the body. At the age of 10 , patient weighed 70 pounds. From this time on the left leg, especially, grew very fast. The mental development of the child had been excellent. She walked and ran like the otler children, but tired very easily. Feet souncled not unlike elephant's feet when walking." Double amputation had beer advised.

Examination.-General condition fair. Patient was thin and had a peaked expression; seemed active and well; pulse slightly rapid at the time of examination. Running down the left arm and leg was a crusty, eczematous lesion, not progressive. The left foot was larger than the right, but both feet were markedly enlarged and lobular in character, the swelling involving the whole of both feet. The same lobulation extended up to the 